\title{
Influence of balnear therapy at Băile Tușnad on quality of life of post-stroke patients
}

Gabriela Dogaru $^{12}$, Alexandra Ispas ${ }^{1}$, Adriana Bulboacă ${ }^{12}$, Marieta Motricală3, Ioana Stănescu' ${ }^{12}$

1.Iuliu Haţieganu" University of Medicine and Pharmacy Cluj-Napoca 2. Clinical Rehabilitation Hospital Cluj-Napoca 3.Tuşnad Spa Complex-Băile Tuşnad

Coresponding author Gabriela Dogaru

E-mail: dogarugabrielaumfci@yahoo.ro

\begin{abstract}
Stroke is a common cause of life-long disability in surviving patients. Despite improvements in neurologic deficits, stroke survivors remain with important limitations on participating in social activities, on reintegrating into family, community and working life which could trigger psychological problems and a decreased quality of life (QoL). An important issue in post-stroke rehabilitation is improvement of patient's quality of life.

Introduction: The assessment of the Quality of Life could be an indicator of the effectiveness of the post-stroke rehabilitation. One simple instrument for assessment of Qol is the Quality of Life Scale (QOLS), created by John Flanagan, which has been adapted for use in chronic illness groups, a higher score indicating higher quality of life. An important instrument for improving QoL of stroke patients is combined rehabilitation therapy in a balneoclimacteric resort or in a rehabilitation hospital.

This study aimed to monitor the efficacy of these natural therapeutic factors, used in conjunction with classical neurorehabilitation methods in post-stroke patients

Material and method: We performed a prospective longitudinal analysis in 50 post-stroke patients which performed rehabilitation treatment: the first group of 20 patients performed only kinesitheraly, massages, occupational therapy during 15 days at Rehabilitation Hospital Cluj, and the second group of 30 patients performed the same treatment during 15 days, in association with with carbonated mineral water baths and walking at Tuşnad Spa Complex SA treatment facility in Băile Tuşnad. Quality of Life of both group of patients was assessed using the QOL Scale.

Results: Both groups showed statistically significant improvements in the QoL as shown by comparing the mean score before and after treatment by the paired $T$ test, with a p value of $<0,05$ in both groups.

Conclusion: Rehabilitation treatment improve the qualty of life in stroke survivors. The use of natural therapeutic factors (mineral water baths and bioclimate), in conjunction with kinesitherapy, occupational therapy and massotherapy, significantly improved the quality of life in stroke patients.
\end{abstract}

Key words: rehabilitation, stroke, carbonated mineral water

\section{INTRODUCTION}

Stroke is a common cause of life-long disability in surviving patients. The impact of stroke on health related quality of life may be disastrous; stroke can affect multiple domains of life. An important outcome in rehabilitation of chronic conditions, such as post-stroke disability, is improvement of patient's quality of life.

Quality of life (QoL) is defined according to World Health Organization as “individual's perception of their position in life in the context of the culture and value system in which they live and in relation to their goals, expectations, standards and concerns" $[1,2]$.
After stabilization of neurological deficits, stroke patients are facing the challenge of adapting their new physical status with their life expectations. This could be a major psychological problem for the patient, and one of the risk factors for poststroke depression. According to different studies, nearly $30 \%$ of stroke patients develop depression, either in the early or in the late stages [3]. In particular, mood depression is considered as the strongest predictor of QoL in stroke survivors [4]. Poststroke depression affects healthrelated QoL, influences functional recovery, cognitive function and healthcare use in stroke survivors. 
Despite improvements in motor deficits or communication problems, stroke survivors remain with important limitations on participating in social activities and on reintegrating into family, community and working life which could trigger psychological problems while facing the new challenges of their post-stroke life. In many stroke survivors, the psychological changes remains unnoticed, making quality of life measurements important factors in assessing the perceptions of health at different time points after stroke and the real impact of stroke in all of the domains of patient's life.

There is a general agreement that QoL is a multidimensional construct that consists of at least three broad domains: physical, mental and social. [5]. The assessment of the Quality of Life could be as well the evaluator of sequelae of stroke as an indicator of the effectiveness of the post-stroke rehabilitation [5]. Quality of Life assessment has been an important part of evaluation of stroke patients and their treatment for more than 30 years [5]. QoL is assessed using specific scales used with three broad domains: physical, mental and social, being a multi-dimensional construct. Most scales are questionnaires based on a patient's subjective self-report or self-evaluation. Some of these tools provide information about perceived health status, for example: physical and mental functions, ability to perform everyday activities. The other scales capture an assessment of well-being or evaluation of particular life domains or satisfaction with life [6].

Health-related quality of life may be influenced by psychological factors, as these factors reflect the way people approach situations and react to stressful situations [7]. Factors of health-related QoL vary with time after stroke onset and reflect the individual's changes of internal standards, values, and expectations influenced by the ongoing recovery process. Psychological determinants of poststroke health-related quality of life are: personality (problems of temperament and personality functions and neuroticism are negatively associated with health-related quality of life), coping (situational and personal adaptation), self-worth (self-esteem and self-efficacy), hope and optimism are all positively associated with healthrelated quality of life [7]. Disability and poststroke depression are other important determinants of QoL in stroke survivors. Other determinants include female sex, coping strategies, and social support [8].

One simple and largely used instrument for assessment of Qol is the Quality of Life Scale (QOLS), created originally by American psychologist John Flanagan in the 1970's, and which has been adapted for use in chronic illness groups. The QOLS is composed of 16 parameters (items) - see Table 1. The QOLS is scored by adding up the score on each item to yield a total score for the instrument. Scores can range from 16 to 112. Each parameter includes a number that indicates the patient's satisfaction/dissatisfaction at that time. The QOLS scores are summed so that a higher score indicates higher quality of life. Average total score for healthy populations is about 90 [9].

\section{Conceptual Category Scale Item}

Material and Physical Material well-being and Well-being financial security Health and personal safety

Relationships with Relations with parents, other People siblings, other relatives Having and raising children

Relations with spouse or significant other Relations with Friends

Social, Community, Activities related to and Civic Activities helping or encouraging others

Activities related to local and national government

Personal Development Fulfillment
Intellectual development and Personal understanding Occupational role 
Conceptual Category Scale Item

Creativity and personal expression

Recreation
Table 1: Flanagan Quality of Life Scale (QOLS) original conceptual categories and scale items [9]

We can also measure health-related QoLmore specifically in stroke patients by using several available and more specific scales, such as: the Burden of Stroke Scale (Doyle, McNeil, Hula and Mikolic 2003), the Stroke Impact Scale (Duncan et al. 1999), the Stroke-Specific Quality of Life Scale (Williams, Weinberger, Harris, Clark and Biller 1999), the Quality of Life Index-Stroke Version (King 1996), the Stroke-Adapted Sickness Impact Profile-30 (van Straten et al. 1997) [10].

Improvement of the quality of life of post-stroke patients implies many aspects. Strengthening of family support, treatment of depression and reduction of physical dependence may be the decisive factors in improving post-stroke QOL [11]. An important instrument for improving QoL of stroke patients is combined therapy in a balneoclimacteric resort [12]. Spa treatment indications must be limited to those patients which showed improvements in their motor function and functional recovery during rehabilitation process, with preserved effort capacity [13] and without unstable neurologic picture or evolving disease aspects.

Balneotherapy is a treatment method that uses natural factors with recognized therapeutic properties based on chemical, mechanical and thermal effects on the organism [14]. Spa therapy is a very popular form of complementary therapy in many countries in Europe [15]. Besides thermal water applications, spa therapy can be combined with a broad spectrum of physical therapeutic modalities including electrotherapy, massage, manipulation, underwater-exercise, ultrasonic therapy, special exercises, cold and hot applications, health education, stress reduction and relaxation.

Spa treatment could be performed either in a classical rehabilitation hospital either in a balneoclimacteric resort with specific natural cure factors. Băile Tuşnad is one of balneoclimacteric areas showing various and specific natural therapeutic factors: mixed carbonated mineral waters, natural mofettes and microclimatic conditions (tonic, stimulating and sparing bioclimate) [12]. The beneficial effects of mixed carbonated waters, mofettes and subalpine bioclimate in Băile Tuşnad are well known and recommended for prevention and rehabilitation treatment in a number of cardiovascular, digestive, renal, nervous system and endocrine diseases.

This study aimed to monitor the efficacy of these natural therapeutic factors, used in conjunction with classical neurorehabilitation methods in post-stroke patients admitted at Tuşnad Spa Complex SA.

\section{MATERIAL AND METHOD}

The study was a prospective longitudinal analysis and was conducted at the Rehabilitation Hospital Cluj and at Tuşnad Spa Complex SA treatment facility in Băile Tușnad.

The study included 50 patients with a history of ischemic or hemorrhagic stroke between april december 2014. The group comprised both female and male patients (19 women and 31 men).

The informed consent of the patients was obtained before their inclusion in the study. The study was approved by the Ethics Committee of the University of Medicine and Pharmacy Cluj-Napoca.

The inclusion criteria in the study were:

- $\quad$ patients with stable neurological deficits after ischemic or hemorrhagic stroke

- $\quad$ at least 6 months after stroke and no more than 18 months after stroke onset

- $\quad$ mild or moderate motor deficit

- $\quad$ patients were able to walk

- $\quad$ stabilized cardiovascular function.

The exclusion criteria were: 
- patients with contraindications of spa treatment (epilepsy, mental disorders, sphincterian troubles)

- patients with contraindications of carbonated mineral water baths.

The patients were included in 2 groups with two different approaches:

1). The first group included 20 patients (5 women and 15 men), aged between 46 and 72 years (mean age 57 years) which attended rehabilitation program in Rehabilitation Hospital in Cluj, during 15 days. Neurorehabilitation methods used were: kinesitherapy 30 minutes daily, for 15 days, massages on specific areas for 15-20 minutes daily - 15 days and occupational therapy - 30 minutes daily for 15 days.

2). The second group included 30 patients (14 women and 16 men), aged between 56 and 89 years (mean age 69 years), which attended rehabilitation therapy at Treatment Facility of the Tuşnad Spa Complex in Băile Tușnad, for 15 days. Treatment methods used by this group consists in carbonated mineral water baths for 15 minutes, daily, for 15 days, aerotherapy and walking for 30 minutes daily during 15 days, kinesitherapy 30 minutes daily - 15 days, massages on specific areas for 15-20 minutes daily - 15 days and occupational therapy - 30 minutes daily for 15 days

Quality of life of each patient was assessed at day 1 , before treatment, and at the end of treatment, on day 15, using the Quality of Life scale.

Data were collected and analyzed using the Microsoft Excel 2007 application. Descriptive statistical analysis was performed in the two groups before and after treatment, with the specific module of the Data Analysis component - Descriptive Statistics. The $\mathrm{T}$ test for the comparison of the means of paired samples was applied for each scale. The statistical significance threshold used was $\mathrm{p}<0.05$.

\section{RESULTS}

1). The first group of patients treated in Rehabilitation Hospital in Cluj showed significant improvement in their QoL as shown by comparing the mean QOLS score before and after treatment by the paired $\mathrm{T}$ test, obtained $\mathrm{p}$ value $<0,05$ $(p=0.000000000000014)$ indicates that the mean after treatment on day 15 (80.35 points) is statistically significantly higher compared to the mean score on day 1 (71 points) (Figure 1).

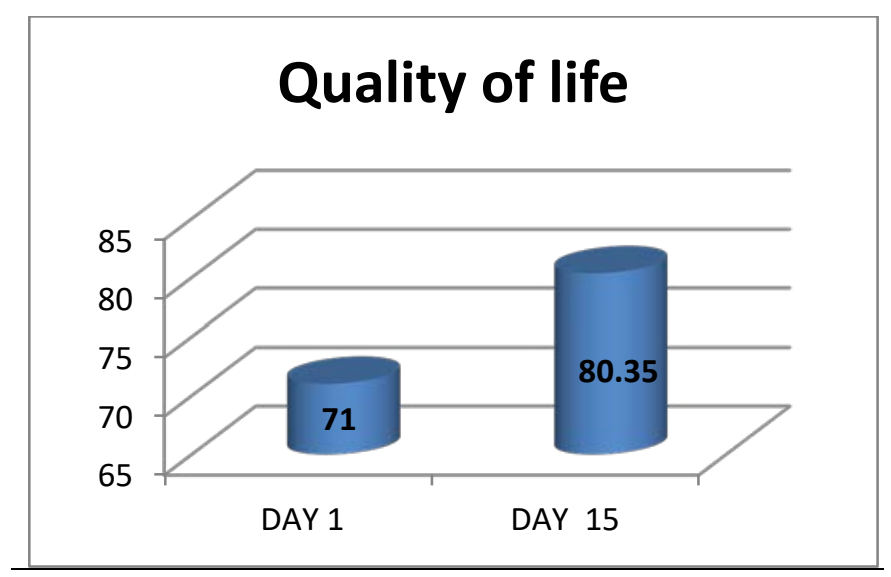

Figure 1. Graphic representation of the means before and after treatment for the QOLS score for group 1

2). The second group of patients were treated in specific natural conditions of Baile Tusnad, in the treatment facility of the Spa Complex, showed also significant improvements in Quality of Life items, as measured by QOLS, with a mean of 77.26 points after treatment compared with 69.56 points before treatment, which is statistically significant: paired $\mathrm{T}$ test $\mathrm{p}<0.05(\mathrm{p}=0.000000000000067$. (Figure 2)

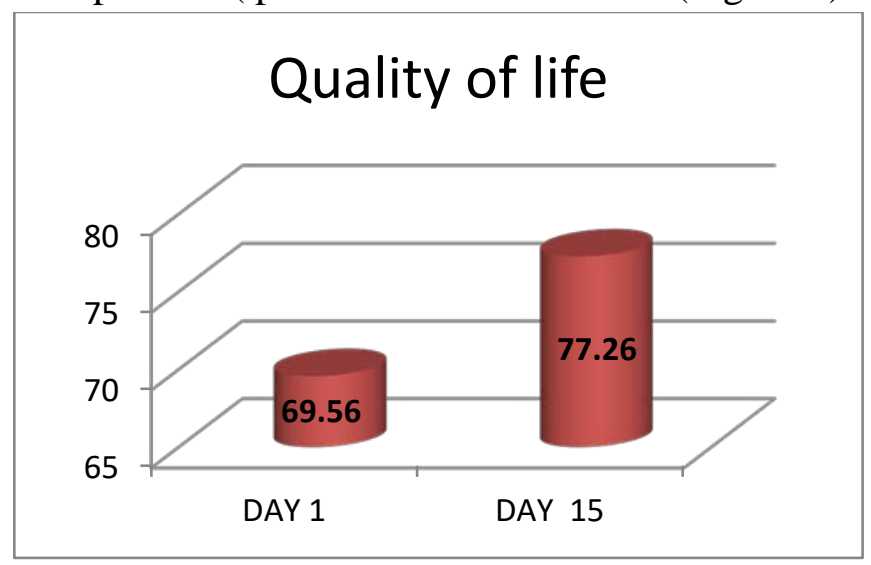

Figure 2: Graphic representation of the means before and after treatment for the QOLS score for group 2 


\section{DISCUSSION}

Improvements in quality of life of post-stroke patients were observed independently of neurologic deficit recovey if the patient was enrolled in a rehabilitation program in a specialized rehabilitation facility. Combined kinesitherapy, regional massages, occupational therapy - even for short periods of time - have a positive impact on perceived health quality of stroke patients, facilitating psychological recovery.

The natural therapeutic factors from a balnear resort (bioclimate, thermal baths) such as Baile Tusnad have synergistic effects with the other methods, improving not only neurologic deficits, but also patient's wellbeing and finally patient's quality of life.

\section{CONCLUSIONS}

The part of clinical study conducted in Rehabilitation Hospital Cluj showed a statistically significant improvement in quality of life of stroke survivors after regular, intensive and structured rehabilitation program, even for a short duration. The part of the clinical study conducted out in Băile Tuşnad Balnear Resort respectively, after statistical processing of the results showed that the use of natural therapeutic factors (i.e. carbonated mineral water and bioclimate through aerotherapy), together with kinesitherapy and massotherapy, significantly improved the quality of life in patients included in the study.

\section{BIBLIOGRAPHY:}

1. WHOQOL Group. The World Health Organization Quality of Life assessment (WHOQOL) position paper from the World Health Organization. Soc. Sci. Med. 1995; 41:1403-1409

2. WHOQOL Group. Study protocol for the World Health Organization project to develop a Quality of Life assessment instrument (WHOQOL). Qual Life Res. 1993;2:153-159.

3. Paolucci S. Epidemiology and treatment of post-stroke depression. Neuropsychiatr Dis Treat. 2008 Feb; 4(1): 145-154

4. Kim P, Warren S, Madill H, Hadley M. Quality of life of stroke survivors. Qual Life Res. 1999 Jun; 8(4):293-301

5. Opara JA, Jaracz K. Quality of life of post-stroke patients and their caregivers. J Med Life. 2010 Aug 15; 3(3): 216-220
6. Carod-Artal FJ. Determining quality of life in stroke survivors. Expert Rev Pharmacoecon Outcomes Res. 2012 Apr;12(2):199-211

7. van Mierlo ML, Schröder C, van Heugten CM, Post MW, de Kort PL, Visser-Meily JM. The influence of psychological factors on health-related quality of life after stroke: a systematic review. Int J Stroke. 2014 Apr;9(3):341-8

8. Carod-Artal FJ, Egido JA. Quality of life after stroke: the importance of a good recovery. Cerebrovasc Dis. 2009;27 Suppl 1:204-14

9. Burckhardt CS, Anderson KL. The Quality of Life Scale (QOLS): Reliability, Validity, and Utilization. Health Qual Life Outcomes. 2003; 1: 60

10. Russell, M., Dempster, M., \& Donnelly, M. Measuring health-related quality of life after stroke: A brief tool. Applied Research in Quality of Life, 2011; 6(1), 41-51.

11. Jaracz K, Kozubski W. Quality of life in stroke patients. Acta Neurol Scand. 2003 May;107(5):324-9.

12. Dogaru Gabriela, Ispas Alexandra, Stanescu Ioana, Motricala Marieta, Ákos Molnar. A clinical study on the efficacy of natural therapeutic factors in Băile Tuşnad for the rehabilitation of post-stroke patients. Balneo Research Journal .2017; Vol.8 (1) :5-10.

13. Frîngu Frangu, Guşetu Gabriel, Iosip Adriana, Gurzău Diana, Dogaru Gabriela, Zdrenghea Dumitru, Pop Dana. The predictors of exercise capacity impairment in diabetic patients. Balneo Research Journal ,2017; Vol.8(2): 26-32.

14. Dogaru Gabriela, Stanescu Ioana, Pop Daniela, Motricală Marieta, Ákos Molnar. Effects of carbonated mineral water treatment in Băile Tuşnad on chronic arterial occlusive disease - a case report. Balneo Research Journal. 2017 ; Vol.8 (3): 121-124

15. Cimbiz Ali, Vahdettin Bayazit, Hasan Hallaceli, Ugur Cavlak. The effect of combined therapy (spa and physical therapy) on pain in various chronic diseases. Complementary Therapies in Medicine. 2005; 13, 244250 